\title{
INDUCTIVE METAL DETECTORS AND THE DESIGN OF PROSPECTING ROBOTS: A POSSIBILITY
}

\author{
Powell Mlambo ${ }^{1}$, Humphrey N Dera ${ }^{1}$, Eugenia R Chiweshe ${ }^{1}$, Enock Jonathan ${ }^{2}$ \\ ${ }^{1}$ Department of Mechatronic Engineering \\ ${ }^{2}$ School of Natural Science and Mathematics \\ Chinhoyi University of Technology \\ Chinhoyi, Zimbabwe \\ pmlambo@gmail.com, humphrey.dera@gmail.com, enyanhete@gmail.com
}

\begin{abstract}
Robots, be they fully or partially autonomous, have the potential to transform and improve the lives of people around the globe by addressing the world's toughest challenges. Robots can be equipped with appropriate sensors to operate in harsh conditions thereby greatly improving the safety of personnel as well as work efficiency, productivity and flexibility. This paper focuses on mobile robotic systems for autonomous/semi-autonomous mineral prospecting in a proficient, reliable, safer and effective way. An overview of inductive metal detectors followed by explanations on the implementation of metal detectors in the design of autonomous/semi- autonomous mobile robotic systems capable of discriminating various metal types is presented here. The paper concludes with possible techniques that can be applied for practical implementation of a new generation of mineral prospecting robots.
\end{abstract}

Keywords: Electromagnetic induction, Autonomous robot, Signal processing, Metal detector, Mineral prospecting

\section{Introduction}

For many years inductive sensors have been applied to different tasks such as distance and approximation sensors in automation control or non-destructive testing and quality testing in search of defects, with most recent researchers focusing on the application of inductive sensing in metal detection [1]. One of the major areas of application of these sensors is in metal detection is for either gold prospecting or demining activities [2], [3, 4] [5]. Important to the mining operations is the ability to identify the position to begin mineral exploitation or activities related to 'gold hunting'. The popularity of metal detectors is increasing with artisanal gold prospectors when investigating extensions or searching for new mineral deposits [6]. Economic viability and a guarantee of ore production over a sufficiently long period of time have to be ensured, before heavy investments required to set up a mining operation are considered. Mobile robotic systems equipped with manipulators and sensors for detecting and locating metals holds much promise in autonomous/semi-autonomous gold prospecting in an efficient, reliable, and effective way [7] [3]. The applied system must be inexpensive, relatively simple and easy to adapt whilst allowing a rapid rate of investment return. This paper thus reviews the most relevant literature and previous research activity relating inductive metal detectors and mobile robots with the main purpose being to help outline the main features, requirements and specifications, for the next generation of mobile robots for autonomous metal detection.

\section{Metal Detectors}

\subsection{Principle of Operation}

Electromagnetic induction (EMI) sensors have been used for the detection of metallic objects for quite a long time and are nowadays considered rather a mature technology [8] [9] [10]. EMI devices, often referred to as "metal detectors", are active, low frequency inductive systems. It must also be noted that EMI sensors still remain an active area of research [8]. The diagram shown in Figure 1 shows a schematic of an EMI device.

ACRID 2017, June 20-21, Victoria Falls, Zimbabwe

Copyright (C) 2017

DOI 10.4108/eai.20-6-2017.2270713 


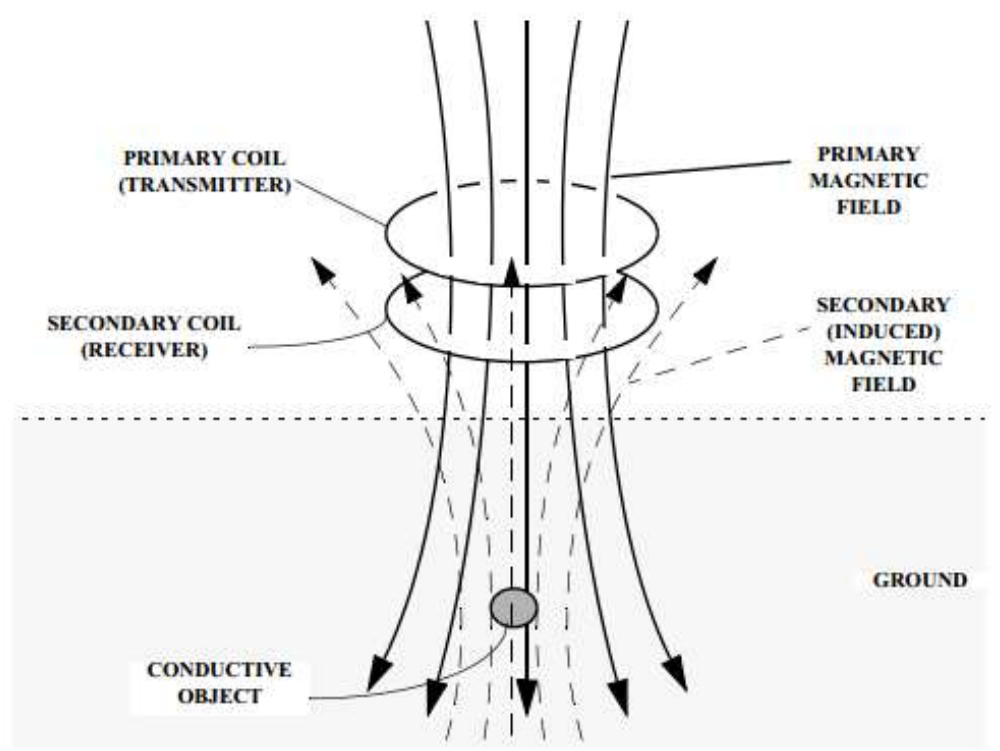

Fig. 1. Schematic of a metal detector

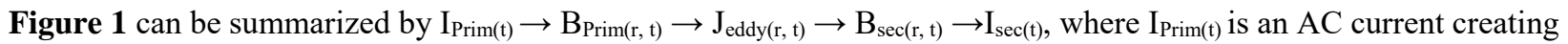
a primary magnetic field, $B_{\text {Prim(r, } t)}$ resulting in eddy currents, $J_{\text {eddy }(r, t)}$ being induced in a metal object which in turn creates the secondary field $B_{\sec (r, t)}$ and its respective current $I_{\sec (t)}$.From Faraday's Law of induction the created eddy current circulate mostly on the surface of the metallic target ("skin effect"), and this explains why metal detectors are mostly surface area detectors [5]. The skin effect states that an electromagnetic field decays in a conducting medium as $\mathrm{e}^{-\mathrm{r} / \delta}$, where $\mathrm{r}$ is the distance from the surface and $\delta$ is a characteristic depth of penetration, the skin depth. In addition the secondary field depends both temporally and spatially, on a large number of parameters of which some are summarized in Figure 2.

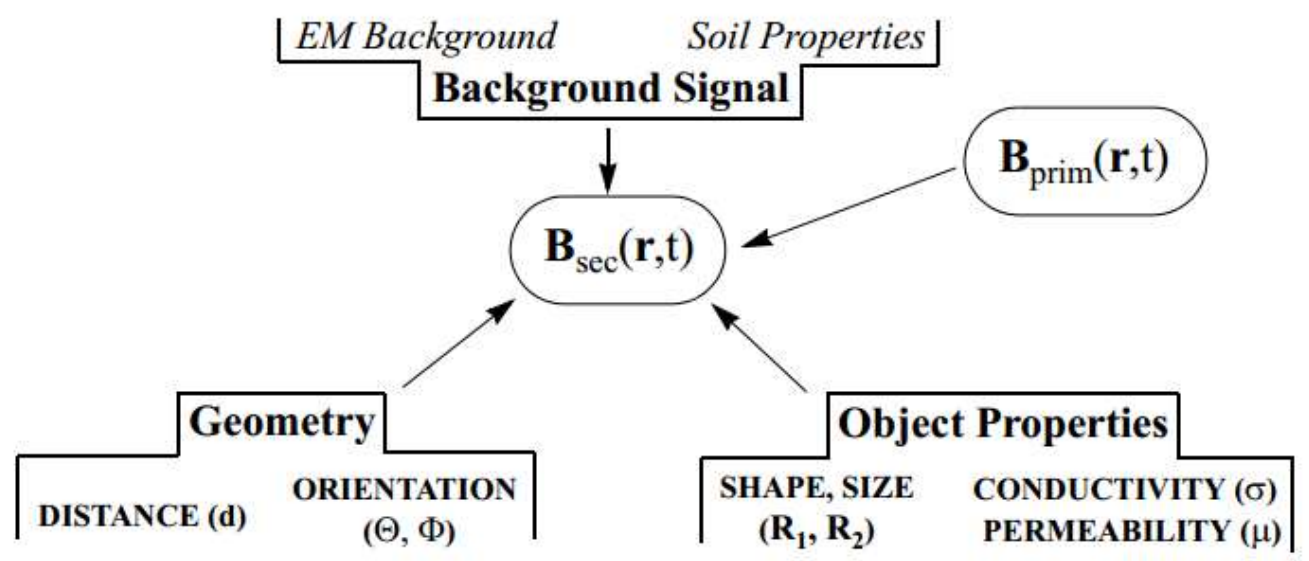

Fig. 2. Parameters influencing induced secondary magnetic fields [5]

From electromagnetism principles derived from Ampere's Law, Maxwell-Faraday equation, Lenz's law and BiotSavart Law an important equation 1 describing magnetic field at a distance $d$ along the axis of the primary coil can be formulated [9].

$$
B=\frac{N \mu_{0} I}{2} \frac{a^{2}}{\left(r^{2}+a^{2}\right)^{\frac{1}{2}}}=\frac{\mu_{0} M}{2 \pi\left(r^{2}+a^{2}\right)^{\frac{1}{2}}} .
$$

, where $N$ is the number of turns of the coil and $M$ is magnetic moment $\left(N i \pi a^{2}\right)$ and $a$ being the radius of a circular coil. From equation 1 it is evident that the magnetic field falls off rapidly with the cube of the distance $d$ from the coil. 


\subsection{Detection Procedure}

In all metal detectors the primary magnetic field must vary in time and this may be achieved either by generating it in the form of short pulses or by varying it smoothly in the form of a discrete number of sinusoidal signals [5]. Therefore EMI sensors may be classified as either Time Domain (TD EMI) or Frequency Domain (FD EMI) sensors. FD EMI devices provide better control over the power and frequency content of the excitation spectrum and generally have higher sensitivity and improved signal to noise response in metal detection applications compared to TD [8].

When considering the detection properties it has been noted that the frequency of the exciting electromagnetic field is an influencing factor, this thus opens the opportunity to develop new methods of primary coil excitation and signal processing of the received signal with intentions of better identifying the target's characteristics. However for a particular application the coil model is usually chosen according to the setting of a task and the coil form and core, with the operating frequencies being determined empirically [10].

For single frequency FD instruments, as the detector approaches a target, the received signal, $V_{s e c}$ can be given as a function of time at the angular frequency $\omega$ of the transmitted signal, but phase shifted (i.e. time delayed) by $\varphi$ as shown in Equation 2 [9] [5]

$$
V_{s e c}(t)=A_{s e c} \sin (\omega t+\varphi) .
$$

Materials that are primarily inductive and excellent conductors show the largest phase shift such as gold, silver, and copper. Smaller phase shifts are typical for objects which are primarily resistive and these are mainly ferromagnetic materials. Expanding Equation 2 results in Equation 3 given as:

$$
V_{s e c}=V_{R} \sin \omega t+V_{X} \cos \omega t .
$$

, where $V_{R}=A_{s e c} \cos \varphi$ and $V_{X}=A_{s e c} \sin \varphi$.

$$
V_{\text {sec }}=V_{R}+i V_{X} .
$$

From Equation 4, the received signal consists of a component which is in phase with the transmitted one (resistive component) and another which is phase shifted (reactive component) or equivalently in the real and imaginary part of the probe's complex impedance change. The discrimination of various metals is thus primarily based on their characteristic phase response.

\subsubsection{Modeling}

To fully understand and describe the system behavior that is - how the detector will respond when a metal is in vicinity, the metal search head can be modeled either analytically or mathematically. The model can then be used for analysis of the signals through simulations and measurements with the intention of finding features that can be used to discriminate various metal types. Simulation software such as COMSOL Multiphysics also provide a good platform for modelling metal detector circuits.

The inductive response of the FD EMI sensor has been established [5]. The target object (Figure 3) is modeled as an isolated conductive circuit with a lumped resistance R and inductance L. The transmitter is labeled "0" and is driven by a current $\mathrm{I}_{0} \mathrm{e}^{\mathrm{i} \omega \mathrm{t}}$, the target circuit is "1", and the receiver circuit "2". The mutual inductances between any two of these circuits are denoted as $\mathrm{M}_{\mathrm{ij}}$, with $\mathrm{i}, \mathrm{j}=0,1,2$ 


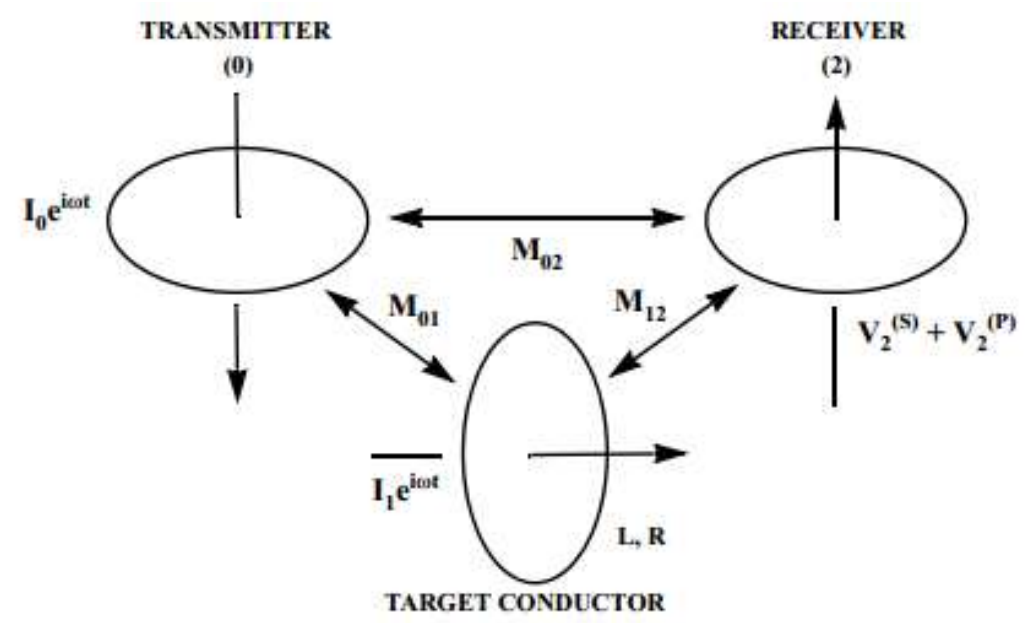

(1)

Fig. 3. A metal detector circuit model

A "response", $\mathrm{G}(\omega)$, can be defined as the ratio between the secondary and primary voltages in the receiver loop, $\mathrm{V}_{2}{ }^{(\mathrm{s})}(\omega) \operatorname{and~}_{2}{ }^{(\mathrm{p})}(\omega)$ respectively.

$$
G(\omega)=\frac{V_{2}^{(s)}(\omega)}{V_{2}^{(p)}(\omega)}=-\frac{M_{01} M_{02}}{M_{02} L}\left[\frac{i \omega L(R-\omega L)}{R^{2}+\omega^{2} L^{2}}\right]=\beta\left[\frac{\alpha^{2}+i \alpha}{1+\alpha^{2}}\right]=\beta\left[\frac{i \alpha}{i+i \alpha}\right] .
$$

with $\alpha=\frac{\omega L}{R}$ and $\beta=-\frac{M_{01} M_{02}}{M_{02} L}$

By studying $G(\omega)$, it can be noted that the coefficient $\beta$ depends only on the relative size and position of the circuits, whereas the term in square brackets depends on the frequency $\omega$ and the target's electromagnetic properties (L, R). From Equation $6 F(\alpha)$, the quasi-magnetostatic transfer function can be extracted .

$$
F(\alpha)=\frac{\alpha^{2}+i \alpha}{1+\alpha^{2}}=\frac{\alpha^{2}}{1+\alpha^{2}}+i \frac{\alpha}{1+\alpha^{2}}=X(\alpha)+i Y(\alpha) .
$$

$X(\alpha)$ is the in-phase component with $Y(\alpha)$ being the quadrature-phase component and "response parameter". Investigations of $F(\alpha)$ shows that its limits exhibits high-pass filtering characteristics:

- As $\alpha \rightarrow \infty X(\alpha) \rightarrow 1$ and $Y(\alpha) \rightarrow 0$ therefore $F(\alpha) \rightarrow 1$. This is denoted as the "inductive limit" which is the case when working at high frequency, or on a highly conductive (low R) or highly inductive target.

- As $\alpha \rightarrow 0, F(\alpha) \rightarrow i \alpha$. This is the "resistive limit", the case when working at low frequency, or on a poorly conductive target (high R).

In summing up, starting from low values of $\alpha$ (resistive limit), the response has initially a small amplitude and is in quadrature. For $\alpha=1$, that is $\omega=R / L$, both components are equal, and for increasing values of $\alpha$ the inductive limit is approached. The phase moves from $90^{\circ}$ at the resistive limit to $0^{\circ}$ at the inductive limit. In a more general sense, the coupling coefficient turns out to be geometry dependent and must be examined separately for each particular model.

\subsubsection{Inverse Electromagnetic Induction Problems}

To recover the position (depth), shape, size and electrical material properties (conductivity and permeability) of a hidden object of finite size the inverse electromagnetic induction problems are introduced. Two general techniques that may be used to estimate the target parameters from measurements are namely "model fitting" and "pattern recognition".

Model fitting involves devising a mathematical model to describe the secondary (induced) fields as a function of source parameters, and then performing maximum-likelihood estimation (such as least squares fitting) to determine the parameter values that best fit the measurements. It is also possible to use a numerical model in the MLE procedure in place of an analytic equation, at the price of increased computational complexity. Using this technique for a 
generalized model given in 2.2.1 if the response function, $G(\omega)$ has been determined, with the mutual inductance between source and receiver and operating frequencies known then what then the parameters that relate to the particular object of interest can be determined. Model fitting is obviously limited by the availability of an applicable model and most geometries do not have simple models.

Patten recognition involves comparing characteristics of a set of electromagnetic data from an unknown object to that of a known object to determine if the two are the same. It also involves analysis of the response curves in the complex plane and their simplification by extracting a corresponding set of features and additional supplementary features. As the number of objects increases large libraries become necessary. Thus the problems becoming less tractable as the libraries increase.

Success of pattern recognition techniques can be cited in landmine detection. A very good example is the curve characterization method shown in Figure 4 developed by [3]. The detector signal was defined in as a 3D representation "Spatially Represented Metal Mine Detector Signal"

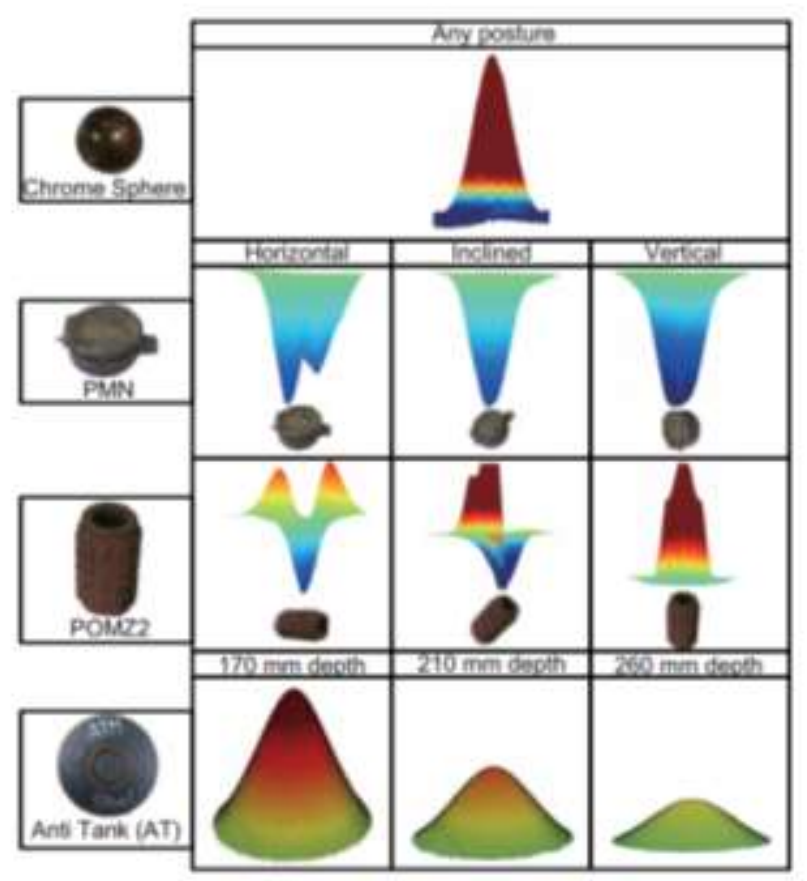

Fig. 4. SRMMDS for different targets at different postures and depths [3]

These approaches though they register a success pose challenges as they rely on a previously built database for discrimination and the discrimination is as limited as the number of data in the database. It is also difficult to build on existing systems and to reuse existing applications.

\section{Robotic Systems}

Inductive metal detectors have found their way mostly in the design of humanitarian demining robots. Most of these systems though being very useful are often expensive, complex and inflexible [11]. The use of reconfigurable systems can come as a solution to some of these problems. The development of a robotic system implies a reliable and cost effective design. Schwertfeger [12] stated that mobile robots are often solely used as mobile platforms for sensors their main task is to bring the sensor where it is needed thus the mobile robot carries the metal detector to the region of interest where the metal searching takes place. 


\subsection{Integration of metal detectors in robots}

Since changes in amplitude and phase of the received signals contain target information, objects are distinguished and identified by their characteristic phase response. However one of the greatest challenges when using the metal detectors is the high levels of false alarms. The use of poly-harmonic signals offers a chance to obtain more complex information about subsurface objects. A model for example developed by Svatoš et al [9], when verified using a Schiebel metal detector with all data processing done in MATLAB, shows that one could differentiate bronze and UNI100Cr6 materials using amplitude and phase spectra analysis as well as Power Spectral Density Periodograms. Signal processing can also be done using other methods such as Wavelet transform and Fast Fourier Transforms so as to better identify the objects. Researches in the following topics also show the potential of automated metal detection [3]:

1. Algorithms for evaluation of detected signals using models of physical phenomena

2. Feature extraction from MMD signals and classification of data according to metal type, size or depth of the metal fragments

It should be noted that a high level of expertise will still be required to properly evaluate the obtained data which is usually an audible sound. Moreover the usual scanning procedure consists of manual sweeps of the detector sideways while advancing the search head in increments between scans. The general procedure given by [16] when using the detectors is as follows:

- Each sweep should overlap the previous sweep by about half the width of the detector

- Sweep rate should be about $30 \mathrm{~cm} / \mathrm{s}$

- The detector should not be more than $5 \mathrm{~cm}$ from the ground

- The detector should remain almost horizontal at all times

There are usually irregularities in the ground, which thus require the operator to constantly reorient the sensor head so as to keep it parallel to the ground. Distractions and fatigue attribute to the operator's inability to maintain the required procedure. A robotic system can follow the same procedure achieving higher precision and repeatability thus increasing the probability of detection.

\subsection{Robotic Manipulators}

Manipulators are employed in mobile robots with the mission of handling sensors and to perform the sweeping/scanning of the interested surface. It's consequently mandatory to design an adaptive scanning of the ground surface to reduce the effect of bad positioning on the useful reflection signal. The manipulator should be designed in such a way that it response to the variations of the terrain ensuring that the sensor head is placed at the appropriate height above the ground and parallel to the ground. A very simple solution for an automated control scheme for adjusting the vertical distance of the sensor head from the terrain is through the use of proximity sensors attached directly to the sensor head, however although technically more complex and expensive, for efficient mapping and scanning of wider areas in a minimal time, cameras and/or laser range finders have to be used Marques, et al [7]. Figure 5 shows a typical passive stereo vision system that works by generating a grid corresponding to the terrain then computes the commands to the actuators for proper positioning of the sensor head. 


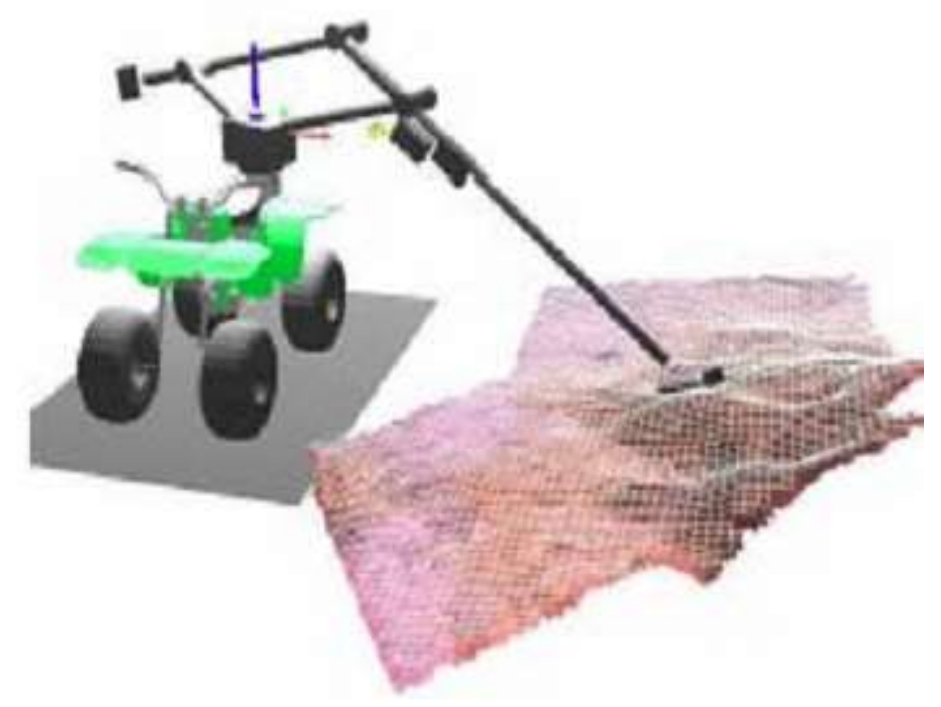

Fig. 5. Robotic Manipulator Stereo System Showing Terrain Mapping [7]

The manipulator should also be designed with considerations being made in terms of its linear speed and distance of line step between scan lines. Kaneko et al [3] points out that the by making use of a robotic manipulator, fast, accurate and high precision scan become possible where there is no need of additional scans.

\section{Discussions}

A number of robotic systems have already been employed which integrates inductive metal detectors [7]. However several of these methods rely on a previously build database for metal discrimination and this is not practical when it comes to detection with the intentions of mineral exploitation. The system architecture for mineral prospecting robots requires the detection system being able to discriminate the minerals of interest from the other minerals present within the ground. A discrimination algorithm that relies heavily on the number of items within a database presents a lot of challenges as mineral nuggets come in an unlimited number of sizes and shapes. Since a number of research has been carried out in regard to feature extraction, recognition strategies applying feature extraction and making decisions based on the available features are possible. Employing narrow band signal processing techniques on receiver signals such as Recursive Gauss Newton method, Forward Amplitude and Phase Estimation, Cross Correlation method, Discrete Fourier transforms or wavelets transforms and integrating this with features from audio signal processing of the generated sound presents ways for pattern recognition and machine learning algorithms.

Once the algorithm has been developed the next stage will be to integrate the inductive metal detectors with a mobile platform in coming up with a prospecting robot, addressing challenges to do with high false alarm and the requirement for high levels of expertise required when using a metal detector. Since the past few decades have also witnessed a lot of research activity in the field of mobile robotics which involves the behavior of robots under dynamic and challenging conditions to achieve a certain goal, the design of a system that can carry out autonomous mineral exploration has been made possible [13]. However the design should allow the robot to operate with some level of autonomy as much as possible receiving assistance whenever necessary. It is important to point out that unlike in landmine detection, there is no need for a mobile platform that withstands explosions therefore light material can be used in the design of the chassis which thus reduces its overall payload and cost. The overall cost of the hardware is greatly important since low capital investments are paramount. The requirements for the prospecting robot can be summarized as shown in table 1 with Figure 6 showing a possible solution concept that can be applied in the design of a prospecting robot. Within the table $\mathrm{D}$ represent requirements which are demands whereas $\mathrm{W}$ depict wishes. 
Table 1.. Requirements for the prospecting robot

\begin{tabular}{|c|c|}
\hline $\mathbf{D} / \mathbf{W}$ & Requirements \\
\hline & Gepmetry \\
\hline D & Length $=400-600 \mathrm{~mm}$ \\
\hline $\mathbf{D}$ & Breath $=300-400 \mathrm{~mm}$ \\
\hline D & Height $=200-250 \mathrm{~mm}$ \\
\hline D & Clearance $=50-70 \mathrm{~mm}$ \\
\hline $\mathbf{W}$ & Wheel Size $180-200 \mathrm{~mm}$ \\
\hline \multirow[t]{2}{*}{$\mathbf{W}$} & Modular Design \\
\hline & Kinematics \\
\hline W & Differential Drive Movement \\
\hline D & Traversable Terrain: Outdoor \\
\hline $\mathbf{W}$ & Max forward/ Backward Movement $=1 \mathrm{~m} / \mathrm{s}$ \\
\hline $\mathbf{W}$ & Max Slope angle 150 \\
\hline $\mathbf{W}$ & Max traversable step $25 \mathrm{~mm}$ \\
\hline $\mathbf{W}$ & Max traversable gap $50 \mathrm{~mm}$ \\
\hline $\mathbf{W}$ & Localization capabilities \\
\hline \multirow[t]{2}{*}{$\mathbf{D}$} & Navigation capabilities \\
\hline & Forces \\
\hline D & Payload $10-15 \mathrm{~kg}$ \\
\hline D & Robot Mass $10 \mathrm{~kg}$ \\
\hline \multirow[t]{2}{*}{$\mathbf{W}$} & Shock absorbers \\
\hline & Energy \\
\hline D & DC powered \\
\hline $\mathbf{W}$ & Runtime $4 \mathrm{hrs}$ \\
\hline W & Charge time $8 \mathrm{hrs}$ \\
\hline \multirow[t]{2}{*}{$\mathbf{W}$} & 12 DC Batteries \\
\hline & Material \\
\hline $\mathbf{W}$ & $1.6 \mathrm{~mm}$ Aluminum \\
\hline $\mathbf{W}$ & Lead Acid batteries \\
\hline W & Temperature $15-600 \mathrm{C}$ \\
\hline \multirow[t]{2}{*}{$\mathbf{W}$} & Humidity $65 \%$ \\
\hline & Signals \\
\hline $\mathbf{D}$ & Metal detection and discrimination \\
\hline $\mathbf{D}$ & No interference between communication equipment and detection system \\
\hline D & Central Control \\
\hline W & Self -Recovery of Robot System \\
\hline \multirow[t]{2}{*}{$\mathbf{W}$} & Modular Control Architecture \\
\hline & Safety \\
\hline D & Operator Safety \\
\hline D & Fail Safe Mechanism \\
\hline \multirow[t]{2}{*}{ D } & Environmental Safety \\
\hline & Ergonomics \\
\hline D & Visual Displays \\
\hline $\mathbf{W}$ & Input Commands Provision \\
\hline W & Human in Loop Provision \\
\hline D & Portable \\
\hline $\mathrm{W}$ & Quality Control \\
\hline $\mathbf{W}$ & $100 \%$ detection Capabilitis \\
\hline \multirow[t]{2}{*}{$\mathbf{D}$} & Detection within a $20 \mathrm{~mm} \times 20 \mathrm{~mm}$ area \\
\hline & Assembly \\
\hline \multirow[t]{2}{*}{$\mathbf{W}$} & Modularized \\
\hline & Maintenance \\
\hline $\mathbf{W}$ & Mean time failure at least a month \\
\hline \multirow[t]{2}{*}{$\mathbf{W}$} & Self-recovery for some problems it may face \\
\hline & Recycling \\
\hline W & Used robot parts should be easily Disposable \\
\hline
\end{tabular}




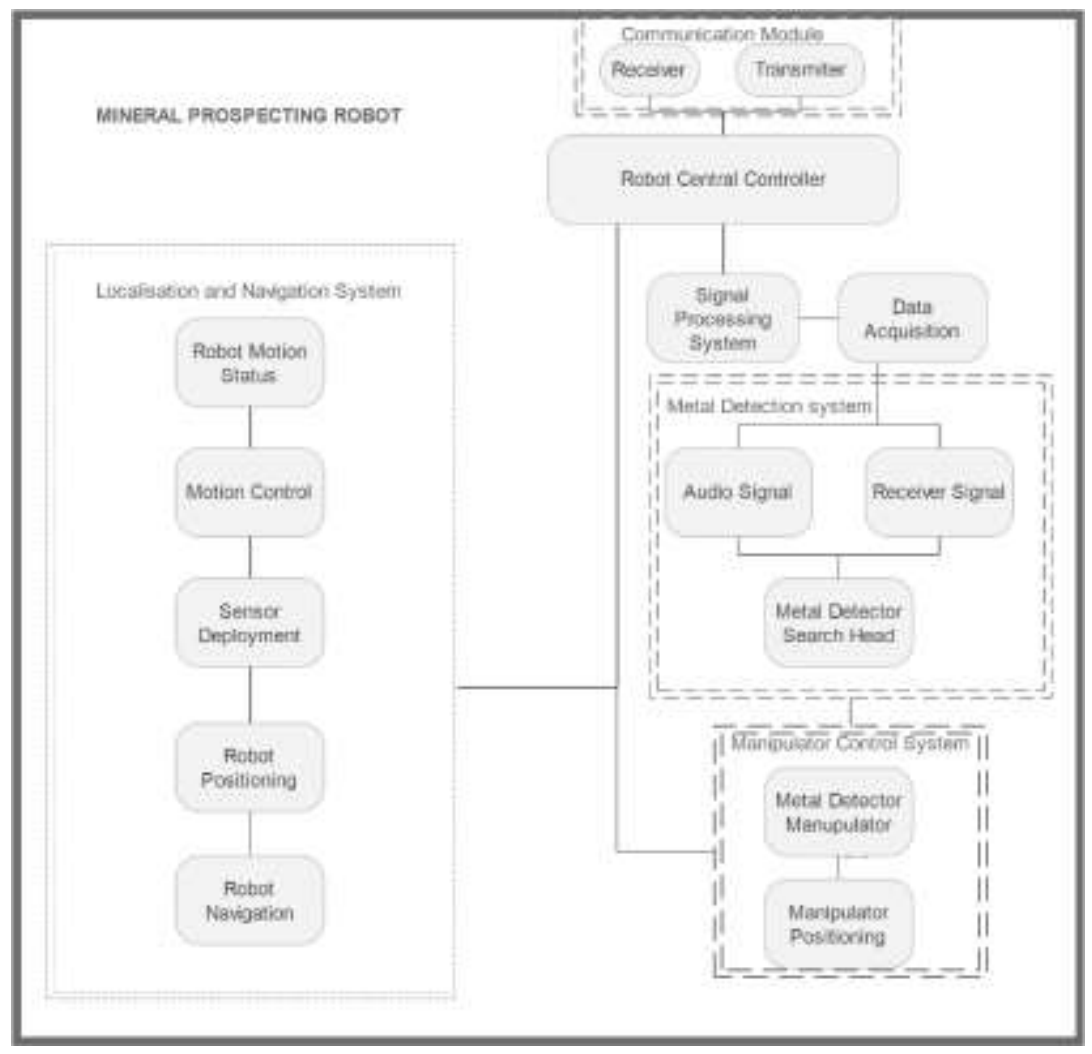

Fig. 6. Solution Concept for a Prospecting Robot

\section{Conclusions}

In this paper the opportunities and challenges that inductive metal detectors provide have been reviewed and the possibility to integrate them in the design of autonomous metal detection systems for the purposes of mineral search exposed. Recognition techniques that can be followed with purposes of metal discrimination have also been analyzed. Application of pattern recognition and machine learning algorithms makes it possible for mineral prospection robots based on inductive metal detectors possible. Further investigations are still required for economic viability in highly unstructured environments.

\section{References}

1. Hartmut E. and Hendrik K.: Inductive sensors and their application in metal detection. In 1st International Conference on Sensing Technology, Palmerston North, New Zealand, 2005.

2. Dave J.: Gold Prospecting with a VLF Metal Detector, 10 Mar 2010.

3. Kaneko A. M., Endo G. and Fukushima E. F.:Landmine Buried Depth Estimation by Curve Characterization of Metal Mine Detector Signals. In International Conference on Intelligent Robots and Systems (IROS), Tokyo, Japan, 2013.

4. Nelson C. V.: Metal Detection and Classifi cation Technologies. In Johns Hopkins APL Technical Digest, vol. Volume 25, Number 1, pp. 62-65, (2004.

5. Bruschini C.: A Multidisciplinary Analysis of Frequency Domain Metal Detectors for Humanitarian Demining. Faculty of Applied sciences, Department Of Electronics And Information Processing, Brussels, 2002.

6. Dessertine A.:From pickaxes to metal detectors: Gold mining mobility and space in Upper Guinea, Guinea Conakry. In The Extractive Industries and Society, vol. 3, no. 2, pp. 435-441, 2016.

7. Marques A. T. d. A. L., Armada M., Fernández R., Montes H., González P. and Baudoin Y.: State of the Art Review on Mobile Robots and Manipulators for Humanitarian Demining. In European Community's Seventh Framework Programme (FP7/20072013), Madrid, 2013.

8. Ambruš D., Vasić D. and Bilas V.: Active induction balance method for metal detector sensing head utilizing transmitterbucking and dual current source. In Sensors \& their Applications XVII , Journal of Physic, IOPscience, 2013. 
9. Svatoš J., Vedral J. and Fexa P.: Metal Detector Excited By Frequency-Swept Signal. In Metrology And Measurement Systems, p. 11, 2011.

10. Ewald H. and Krüger H.: Inductive sensors and their application in metal detection. In 1st International Conference on Sensing Technology, Palmerston North, New Zealand, 2005.

11. David P, Cabrita G, Bruno G and David C.: An autonomous all terrain robotic system for field demining missions. In Robotics and Autonomous Systems, ELSERVIER, vol. 70, pp. 126-144, 2015.

12. Schwertfeger S.: Robotic Mapping in the Real World: Performance Evaluation and System Integration.. Bremen, 2012.

13. Leena.N. and Saju K.: A survey on path planning techniques for autonomous mobile robots. In IOSR Journal of Mechanical and Civil Engineering (IOSR-JMCE), Vols. 2278-1684, no. 2320-334X, pp. 76-79, 2014.

14. Atlas-Copco.: Prospecting and exploration. In Technically speaking, pp. 14-19, 2003.

15. Bruce C.: Metal Detector Metal Detector," 2007.

16. Roemi ., Héctor M., Carlota S., Santos P. G. and Armada M., "Design o fa training tool for improving the use of hand-held detectors in humanitarian demining," Panama, 2013. 